\title{
Polycyclic Phosphiranes: Synthesis of C-Substituted BABAR-Phos Compounds
}

\author{
Florian B. Läng* and Hansjörg Grützmacher
}

\begin{abstract}
BABAR-Phos is a very stable polycyclic phosphirane which is easily synthesized in few steps from dibenzosuberenone. BABAR-Phos is remarkably stable and is not oxygenated with $\mathrm{O}_{2}$ nor does it react with sulfur in boiling toluene. BABAR-Phos can be used as a ligand in homogenous catalysis. Substituents at the carbon of the $\mathrm{PC}_{2}$ heterocycle can be introduced and asymmetric BABAR-Phos were prepared. The coordination chemistry of rhodium complexes containing these as ligands was investigated.
\end{abstract}

Keywords: Coordination chemistry · Phosphanes · Phosphiranes · Rhodium

\section{Introduction}

Phosphiranes are three-membered rings containing a strained $\mathrm{PC}_{2}$ heterocycle. Various methods for their synthesis have been developed and their reactivity has been explored [1][2]. A typical property is their tendency to undergo [2+1] cycloreversions which has been used to generate phosphinidenes, R-P:, as reactive intermediates. Frequently, this reaction is even facilitated in phosphirane complexes and phosphinidene complexes, $\left[\mathrm{M}(=\mathrm{PR}) \mathrm{L}_{\mathrm{n}}\right]$, are obtained. However, this decomposition impedes the use of phosphiranes as ligands. We therefore embedded the heterocyclic $\mathrm{PC}_{2}$ unit into a polycyclic framework and obtained very stable phosphiranes $\mathbf{5}$, which we named BABAR-Phos [3]. These compounds can be handled and stored in air without decomposition and are not sensitive against sulfurization with elemental sulfur in boiling toluene or strong alkylating agents like $\mathrm{MeOSO}_{2} \mathrm{CF}_{3}$. They also resist aqueous acids or bases. However, degradation is observed when solutions of BABAR-Phos in halogenated solvents are exposed to light and air for longer periods of time.

\section{Synthesis}

The synthesis of BABAR-Phos starts from the commercially available dibenzosuberenone $\mathbf{1}$ which is converted via the chloride $\mathbf{2}$ and amine $\mathbf{3}$ to the amino(dichloro)phosphanes 4 (Scheme 1). Dehalogenation of the $\mathrm{PCl}_{2}$ compounds $\mathbf{4}$ with magnesium turnings in THF leads to formation of the phosphiranes 5. This new method most likely proceeds via (amino)- phosphinidene magnesium complexes of the type $[\mathrm{R}$ (trop) N-P=Mg] [4] which undergo an intramolecular cycloaddition with the $\mathrm{C}=\mathrm{C}$ double bond of the seven-membered ring of the trop unit (trop $=5 \mathrm{H}$-dibenzo[a,d]cyclohepten-5-yl). Due to the spatial proximity of the phosphinide and olefinic unit within the rigid boat conformation of the dibenzotropylidene unit, this key-step is favored. The reaction proceeds at room temperature with excellent yields (>90\%).

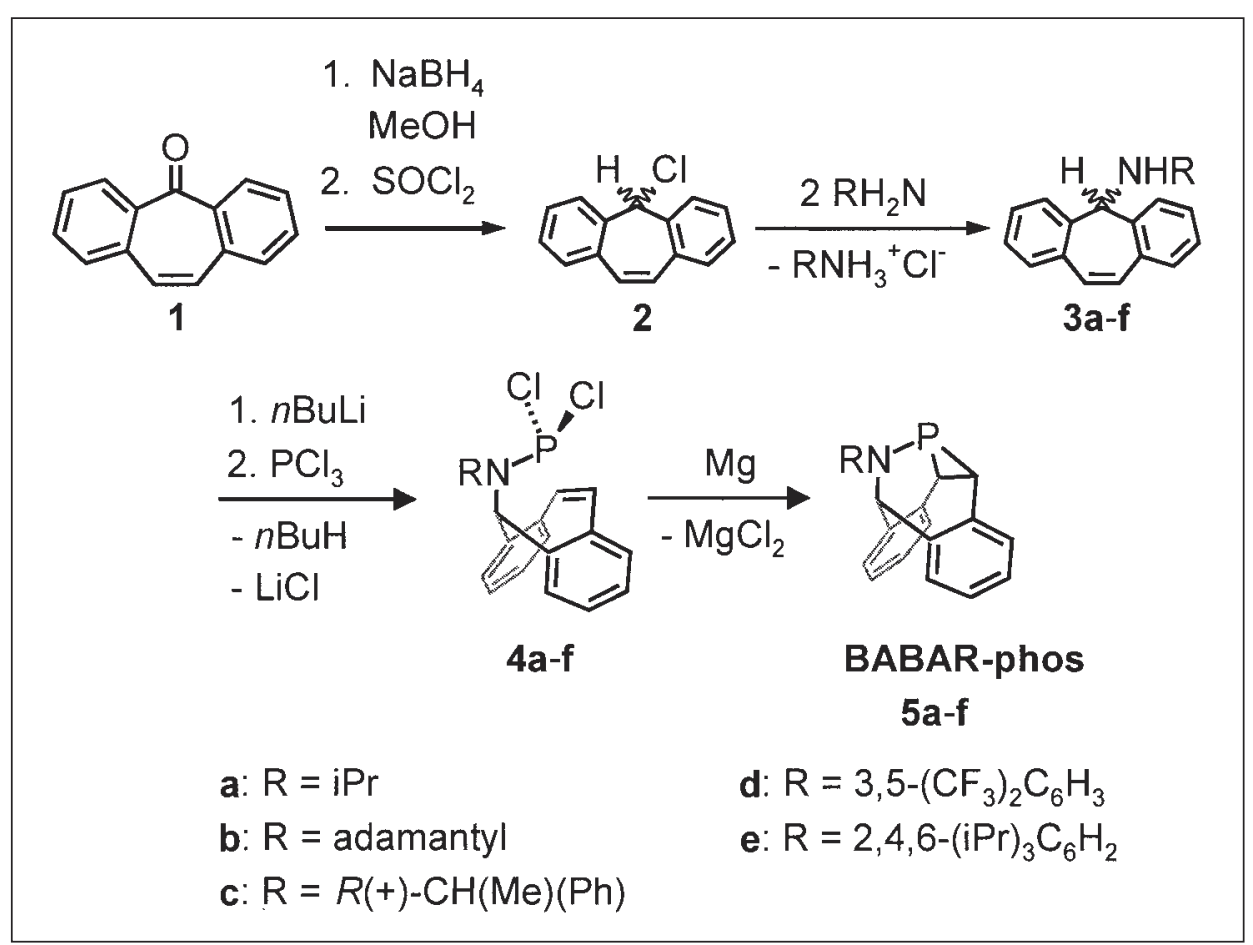


Due to the strong pyramidalization at the phosphorus center $\left[\Sigma^{\circ}(\mathrm{P}) 240-250^{\circ}\right]$, one might expect that BABAR-Phos behaves as a $\pi$-acceptor but also as a rather poor $\sigma$-donor because the donor-orbital (HOMO) at the phosphorous has a high s-character and is strongly contracted [5]. In a recent thermochemical study combined with DFT calculations, we could show that BABAR-Phos is the most weakly binding phosphane known to date in the series $\left[\mathrm{Pt}(\mathrm{cod}) \mathrm{Me}_{2}\right]+2 \mathrm{PR}_{3} \rightarrow\left[\mathrm{PtMe}_{2}\left(\mathrm{PR}_{3}\right)_{2}\right]+$ cod; with $\mathrm{PR}_{3}=$ BABAR-Phos: $\Delta \mathrm{H}_{\mathrm{r}}=$ $-11.8 \mathrm{kcal} / \mathrm{mol}$, [6]. We also found that metal centers can be reversibly inserted into one of the $\mathrm{P}-\mathrm{C}$ bonds of the $\mathrm{PC}_{2}$ cycle [7]. This equilibrium, metal BABAR-Phos complex $\leftrightarrows$ metallaphosphetane, is very sensitive with respect to the coordinating metal center and the electronic properties of the further co-ligands. With both the complexes containing the intact BABARPhos ligand as well as with the metallaphosphetanes, we could perform promising hydroboration and hydrosilylation experiments [6-8].

\section{Results and Discussion}

To further investigate the properties of BABAR-Phos as a ligand, we started to introduce various substituents $\mathrm{R}$ into the polycyclic framework. Evidently, changing the N-bonded group $\mathrm{R}$ is simple and experimentally realized using different sufficiently bulky primary amines in the reaction with the chloride 2 (see Scheme 1 for with $\mathrm{R}=$ alkyl or arene). However, the reaction presented in Scheme 1 is not practical with small amines (i.e. $\mathrm{MeNH}_{2}$ ), as one ends up with the expected mixture of di- and tri(alkylated) amines. In this case, tropolone $\mathbf{1}$ is converted to the corresponding imine 6 which can be reduced with $\mathrm{NaBH}_{4}$ to the desired amine $\mathbf{3 f}$ (Scheme 2).

Although the N-bonded substituent has a significant influence on the activity and selectivity of the catalyst in hydroborations [9] a chiral group as in $\mathbf{5 c}$ gave disappointingly no enantioselectivity. We therefore thought to introduce one substituent at the $\mathrm{C}$-atom of the $\mathrm{PC}_{2}$ heterocycle which also would lead to a break-down of the mirror symmetry of BABAR-Phos.

To functionalize the $\mathrm{C}$-atom of the heterocycle, we prepared the 10-bromo substituted dibenzocycloheptenol 9 which is easily accessible in three steps from dibenzosuberenone 1 via bromination to give $\mathbf{7}$, dehydrobromination to give $\mathbf{8}$, and reduction of ketone $\mathbf{8}$ with $\mathrm{NaBH}_{4}$ in almost quantitative yield (Scheme 3).

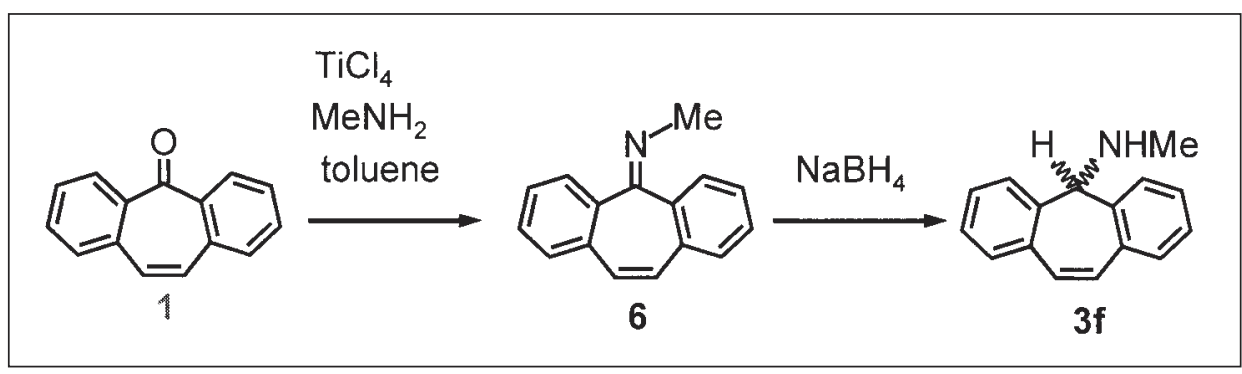

Scheme 2. Synthesis of the sterically less demanding tropamine $\mathbf{3 f}$ as precursor for a $\mathrm{N}$-methyl BABAR-Phos $5 f$.

The alcohol 9 turned out to be a versatile reagent in palladium-catalyzed Suzuki cross coupling reactions [10]. The reaction with arylboronic acids, $\operatorname{ArB}(\mathrm{OH})_{2}(\mathrm{Ar}=$ aryl $=$ phenyl, naphtyl, anisyl) provides the $\mathrm{C}$-substituted alcohols $\mathbf{1 0 a}-\mathbf{c}$ in very good yields $(>80 \%)$.

The C-substituted alcohols 10a-c were subsequently converted to the corresponding chlorides and further transformed into the final iPr-BABAR ${ }^{A r}-P h o s ~ 11 a-c$ according to Scheme 1 (in our notation, the $\mathrm{N}$-bonded substituent is given as prefix and the C-bonded one as superscript at the ter- minus). The phosphirane ring was successfully formed with all aryl substituents. However, with the rather bulky 1-naphtyl substituent side products were observed, presumably polyphosphanes, because the $\mathrm{C}=\mathrm{C}_{\text {trop }}$ double bond is less accessible. The observed ${ }^{31} \mathrm{P}$ chemical shifts of the $\mathrm{C}$-substituted phosphiranes are in the range of -128 to $-132 \mathrm{ppm}$. Note that free rotation of the 1-napthyl and the 2-anisyl substituents in the phosphiranes $\mathbf{1 1 b}, \mathbf{c}$ is frozen on the NMR time scale and hence two ${ }^{31} \mathrm{P}$ NMR signals are observed, each indicating one rotational isomer.

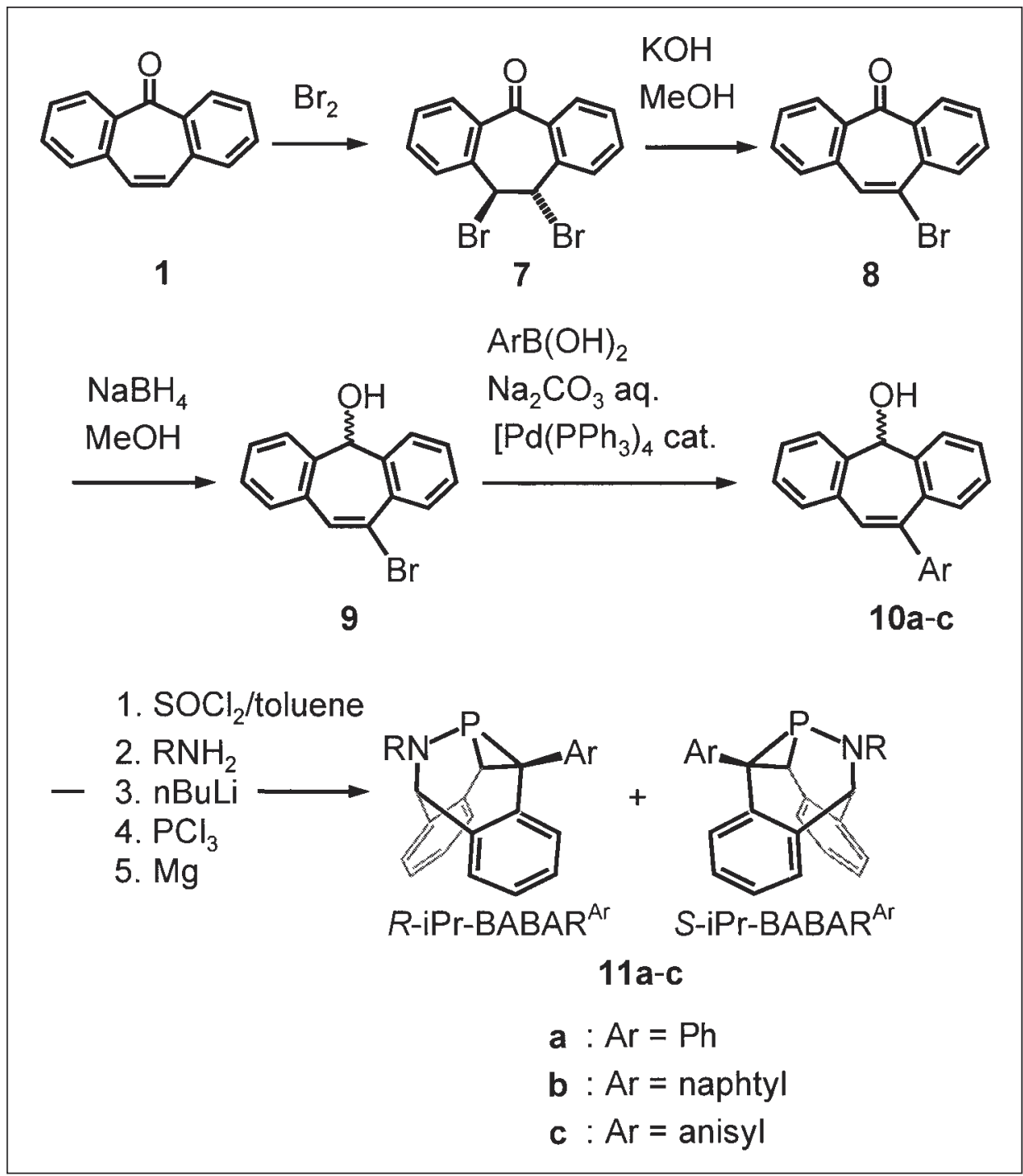

Scheme 3. Synthesis of the iPr-BABAR ${ }^{\text {Ar-Phos } 11 a-c}$ 
In these reactions, the phosphiranes 11a-c are obtained as a racemic mixture of both enantiomers (see Scheme 4 where we denoted the stereochemistry at each phosphorus center as $(R)$ or $(S)$, respectively). We introduced an additional stereogenic center at the $\mathrm{N}$-substituent in the hope of being able to eventually separate the diastereomers. This approach was realized by transforming the alcohol 10a into the corresponding chloride and then reacting this with enantiomeric pure $(R)-(+)-1-$ phenylethylamine. Unfortunately, however, we were not able to separate the finally obtained diastereomeric phosphiranes by chromatography.

We started to investigate the coordination chemistry using the $\mathrm{C}$-substituted phosphiranes 11a-c as ligands for rhodium complexes. When the racemic mixture of $(R / S)$-iPr-BABAR ${ }^{\mathrm{Ph}}$ 11a was reacted with $\left[\mathrm{Rh}_{2}\left(\mu_{2}-\mathrm{Cl}\right)_{2}(\operatorname{cod})_{2}\right]$ in acetonitrile as solvent, independently of the molar ratios of the reactants, the $\mathrm{Rh}(\mathrm{I})$ complex 12a was obtained exclusively as a brown powder which rapidly precipitates out of the reaction mixture.

The isolated complex consists of a $\mathrm{Rh}(\mathrm{I})$ center which is coordinated by one chloro and three $\mathrm{iPr}-\mathrm{BABAR}{ }^{\mathrm{Ph}}$ ligands 11a. Using two-dimensional NMR methods, the structure was determined to be the $(R, R, S)$ isomer and the enantiomeric $(S, S, R)$-form, respectively, as is shown in Scheme 4. When this isolated complex is re-dissolved in THF, complicated ${ }^{31} \mathrm{P}$ NMR spectra are obtained which slowly evolve with time. We assign these to a number of different conformational and rotational isomers formed slowly in equilibria involving complexes of type 12a. Each of these species shows three inequivalent ${ }^{31} \mathrm{P}$ resonances and each of them is split into an eight-line pattern (ddd) by coupling with two ${ }^{31} \mathrm{P}$ nuclei and one ${ }^{103} \mathrm{Rh}$ nucleus. Finally, on storing a THF solution of 11a layered with n-hexane over two weeks at room temperature, single crystals of 12a were obtained. The result of an X-ray structure analysis is given in Fig. 1, which shows this isomer to have an $(R, R, R)$-conformation (the $(S, S, S)$-enantiomer, which is also included in the crystal lattice, is not shown). Note, however, that also these all- $(R)$-, respectively, all- $(S)$-isomers show three different ${ }^{31} \mathrm{P}$ resonances indicating hindered rotation around the $\mathrm{P}-\mathrm{Rh}$ bonds. The otherwise slow rate of the isomerization of the initially formed kinetic product 12a with $(R, R, S / S, S, R)$-conformation can be increased to minutes when AgOTf is added to the THF solution.

As the free phosphiranes, the complexes 12a show an unusually high robustness against oxidation and are not decomposed

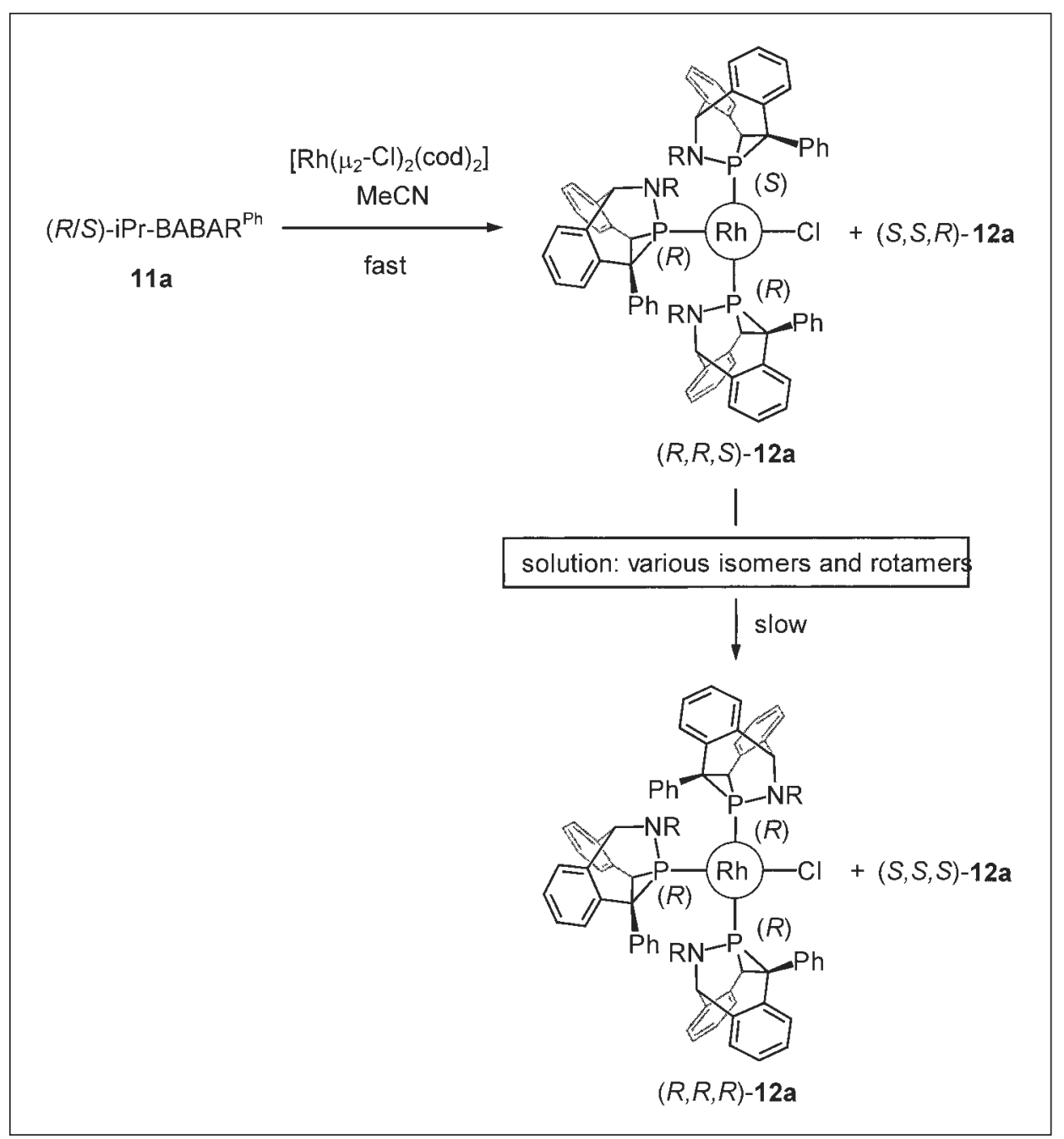

Scheme 4. Synthesis of rhodium(I) complexes 12a with the racemic mixture of 11a.

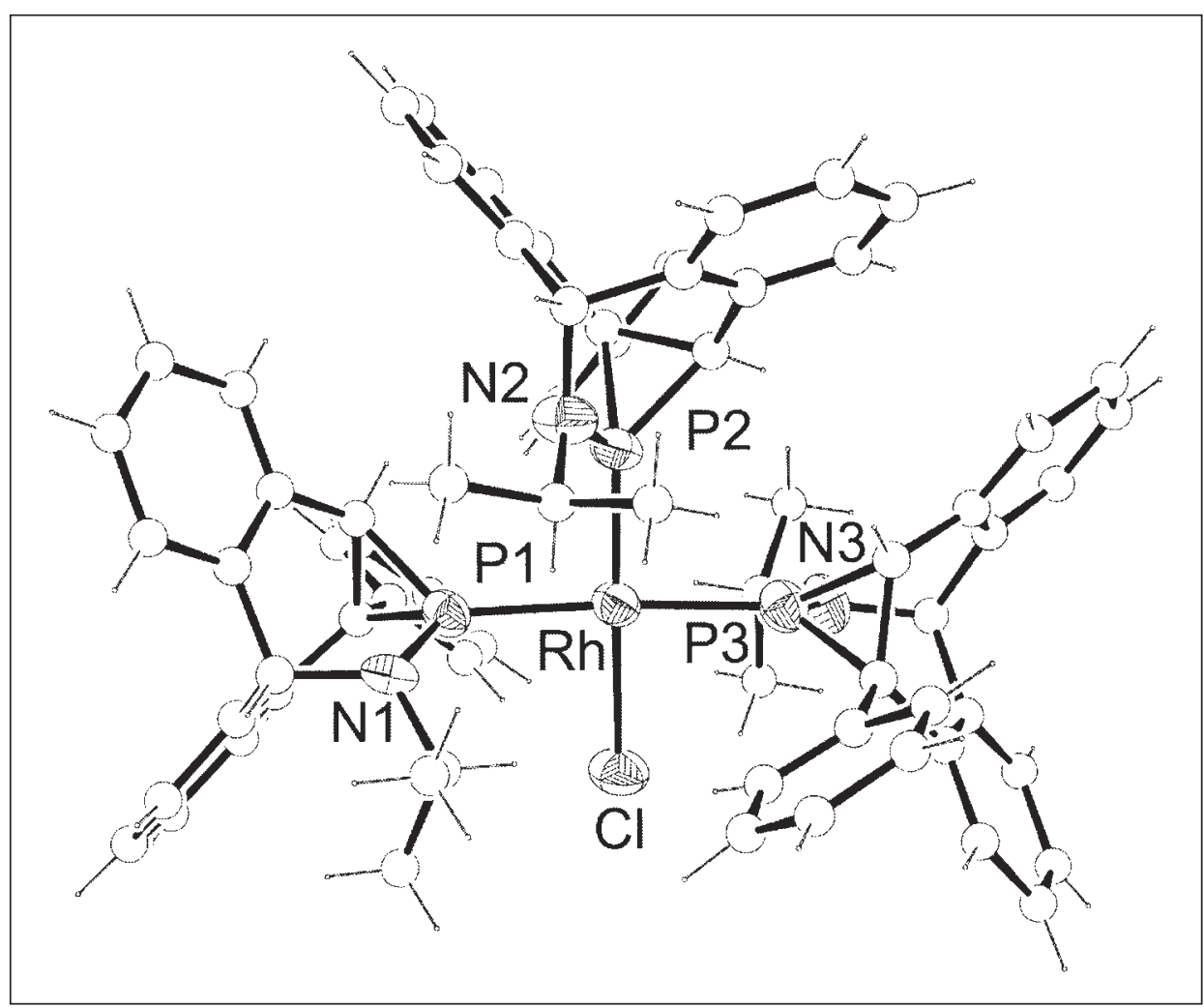

Fig. 1. Structure of the $(R, R, R)$-isomer of $\left[\mathrm{RhCl}\left(\mathrm{PPr}-\mathrm{BABAR}{ }^{\mathrm{Ph}}\right)_{3}\right] \mathbf{1 2 a}$. 
even under $5 \mathrm{~atm}$. of pure oxygen in the solid state. In accordance with the results from the calorimetry experiments cited above [6], in solution the complexes 12a are rather labile and the $\mathrm{iPr}-\mathrm{BABAR}^{\mathrm{Ph}}$ Phos ligands are quantitatively displaced when stronger ligands such as $\mathrm{PPh}_{3}$ or $\mathrm{P}(\mathrm{OMe})_{3}$ are added. Interestingly, while we could observe complexes with a heteroleptic coordination sphere when $\left[\mathrm{PtMe}_{2}(\mathrm{iPr}-\right.$ $\mathrm{BABAR})_{2}$ ] was reacted with phosphanes, $\mathrm{PR}_{3}$, comparable complexes, i.e. [RhCl(iPr$\left.\left.\mathrm{BABAR}^{\mathrm{Ph}}\right)_{3{ }^{-} \mathrm{x}}\left(\mathrm{PR}_{3}\right)_{\mathrm{x}}\right]$, were not observed. Only the homoleptic complexes 12a beside $\left[\mathrm{RhCl}\left(\mathrm{PR}_{3}\right)_{\mathrm{x}}\right](\mathrm{x}=2,3)$ and free $\mathrm{PPr}-\mathrm{BABAR}{ }^{\mathrm{Ph}}$ were detected. This finding together with the observation that in the syntheses of rhodium(I) BABAR-phos complexes frequently the per-substituted complexes $\left[\mathrm{Rh}(\mathrm{BABAR}-\mathrm{Phos})_{4}\right]^{+}$or $[\mathrm{RhCl}(\mathrm{BABAR}-$ Phos $\left.)_{3}\right]$ are formed even when a less than stoichiometric amount of ligand is used, leads us to suspect that additional van-derWaals interactions within the ligand sphere augment the stability of the products. This aspect as well as catalytic reactions with the BABAR-Phos compounds described here are under current investigation.

Received: February 7, 2003
[1] F. Mathey, 'Phosphorus-Carbon Heterocyclic Chemistry: The Rise of a New Domain', Pregamon Press, Amsterdam, 2001.

[2] K.B. Dillon, F. Mathey, J.F. Nixon, 'Phosphorous: The Carbon Copy', Wiley, New York, 1998.

[3] J. Liedtke, S. Loss, G. Alcaraz, V. Gramlich, H. Grützmacher, Angew. Chem. 1999, 114, 1724, Angew. Chem. Int. Ed. Engl. 1999, 38, 1623. The name BABAR-Phos is a symbiosis out of the related hydrocarbon barbaralane and the obvious resemblance of BABAR-Phos to the famous green elephant from the children's books (see sketch below).

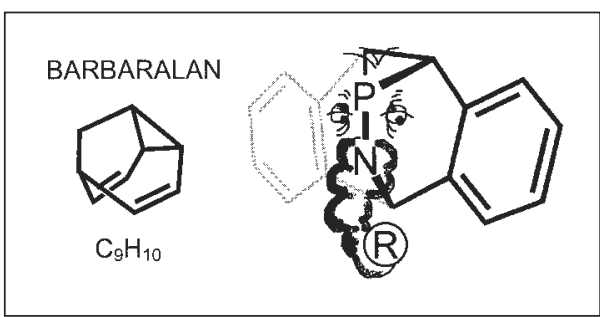

Fig. 2. Structure of barbarlane, $\mathrm{C}_{9} \mathrm{H}_{10}$, and BABAR-Phos the 'elephant'.

[4] H. Bock, M.Bankmann, J. Chem. Soc., Chem. Commun. 1989, 1130.

[5] B.J. Dunne, R.B. Morris, A.G. Orpen, J. Chem. Soc. Dalton Trans. 1991, 635.
[6] C. Laporte, G. Frison, H. Grützmacher, A.C. Hillier, W. Sommer, S.P. Nolan, Organometallics, in press.

[7] J. Liedtke, H. Rüegger, S. Loss, H. Grützmacher, Angew. Chem. 2000, 112, 2596, Angew. Chem. Int. Ed. Engl. 2000, 39, 2479.

[8] J. Liedtke, S. Loss, H. Grützmacher, Tetrahedron (Symposium in Print) 2000, 56, 143.

[9] H. Grützmacher, J. Liedtke, G. Frasca, F. Läng, N. Pè, Phosphorous, Sulfur and Silicon 2002, 177, 1771.

[10] F. Läng, unpublished results of the planned $\mathrm{PhD}$ thesis.

\title{
Stabilization of Molecular LiF, LiFHF, and $\mathrm{Na}_{2} \mathrm{SiF}_{6}$ Using Metallamacrocyclic Hosts
}

\author{
Marie-Line Lehaire* and Kay Severin
}

\begin{abstract}
The molecular forms of LiF, LiFHF and $\mathrm{Na}_{2} \mathrm{SiF}_{6}$ have been stabilized using trinuclear metallamacrocyclic complexes of (cymene) $\mathrm{Ru}^{\prime \prime},\left(\mathrm{Cp}^{*}\right) \mathrm{Rh}^{\mathrm{III}}$ and $\left(\mathrm{Cp}^{*}\right) \mathrm{r}^{\prime \prime \prime}$ as specific receptors. The host-guest complexes were characterized by NMR spectroscopy and single crystal X-ray diffraction. Based on these results, a highly selective chemosensor for fluoride anions has been developed.
\end{abstract}

Keywords: Chemosensor · Fluoride · Lithium · Metallamacrocycle · Sodium · Stabilization

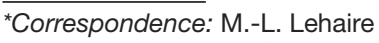

Institut de Chimie Moléculaire et Biologique École Polytechnique Fédérale de Lausanne

$\mathrm{CH}-1015$ Lausanne

Tel.: + 41216939313

Fax: + 41216939305

E-Mail: marie-line.lehaire@epfl.ch

\section{Introduction}

Recently, we have investigated the selfassembly of (cymene) $\mathrm{Ru}^{\mathrm{II}},\left(\mathrm{Cp}^{*}\right) \mathrm{Rh}^{\mathrm{III}}$ and (Cp*)IrII complexes using 3-hydroxy-2pyridone as the bridging ligand [1-3]. Trinuclear metallamacrocycles were obtained in all cases. They possess three oxygen donor atoms positioned in close proximity to each other and can thus be considered as organometallic analogues of 12-crown-3 (Scheme). The metallacrown complexes were attested to be powerful ionophores with outstanding affinities toward $\mathrm{Na}^{+}$ 